\title{
Saúde da Mulher, Gênero, Políticas Públicas e Educação Médica: Agravos no Contexto de Pandemia
}

\author{
Women's Health, Gender, Public Policies and Medical Education: Issues in the Context of the Pandemic
}

Verônica Clemente Ferreira' (D, Mariana Regazzi Ferreira da Silva" (D), Elisa Hypólito Montovani" (D)

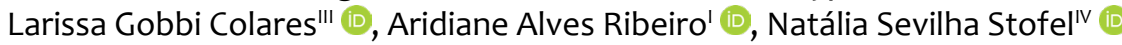

\begin{abstract}
RESUMO
Introdução: A pandemia de Covid-19 agrava as desigualdades sociais e torna urgente olhar para as populações vulneráveis, especialmente as mulheres. $O$ isolamento social e a crise econômica intensificam a violência contra as mulheres e dificultam seu acesso à saúde.

Desenvolvimento: $O$ reconhecimento das vulnerabilidades sociais desse grupo na pandemia reitera a necessidade de uma formação médica atenta às desigualdades de gênero e alinhada às políticas públicas de saúde da mulher no SUS. Este ensaio tem como objetivo refletir sobre as competências necessárias aos graduandos em Medicina, buscando uma atenção integral à saúde da mulher e em diálogo com as políticas públicas vigentes e as DCN. Este texto está organizado em quatro áreas: saúde materno-infantil, saúde sexual, direitos reprodutivos e cuidados com mulheres no climatério e na menopausa.
\end{abstract}

Conclusão: Observamos que, para garantir a saúde integral da mulher, as escolas médicas devem dar aos alunos oportunidades de aprender um conjunto de habilidades, para que, uma vez formados, possam: pautar sua conduta por evidências científicas, ouvir as mulheres, comunicar-se adequadamente com elas, respeitar suas singularidades em cada etapa do ciclo ginecológico, construir uma relação mais simétrica, adotar uma visão ampla de suas condições de vida e dar à mulher maior controle sobre o próprio corpo, a saúde, sexualidade e vida.

Palavras-chave: Covid-19; Epidemia por Novo Coronavírus (2019-nCoV); Saúde Feminina; Saúde das Mulheres; Educação Médica.

\section{ABSTRACT}

Introduction: The COVID-19 pandemic has exacerbated social inequalities and further exposed the vulnerability of certain groups in society, especially women. Social isolation measures and the ensuing economic crisis have led to women suffering even greater levels of violence and more limited access to health.

Development: The recognition of women's social vulnerabilities during the pandemic reinforces the need for a medical education that takes heed of gender inequalities and is aligned to the public policies on Women's Health in the SUS. This essay aims to reflect on the skills needed by medical undergraduates, with a view to achieving comprehensive health care for women and in dialogue with current public policies and national curriculum guidelines. The study was organized in four areas: Maternal and Child Health; Sexual Health; Reproductive Rights and Care for Women in Perimenopause and Menopause.

Conclusion: We observed that to ensure comprehensive women's health care, medical schools should give to students the opportunities to learn a skill set that will prepare them to: guide their conduct by scientific evidence, listen to women, communicate properly with them, respect their particular characteristics in each stage of the gynecological cycle, build more symmetrical relationships, adopt a broad view of their living conditions and give women greater control over their bodies, health, sexuality and life.

Keywords: Covid-19; New Coronavirus Epidemic (2019-nCoV); Women's Health; Medical Education.

'Universidade Federal de Jataí, Jataí, Goiás, Brasil.

"Universidade do Estado do Rio de Janeiro, Rio de Janeiro, Rio de Janeiro, Brasil.

"'Centro Universitário de Belo Horizonte, Belo Horizonte, Minas Gerais, Brasil.

"Universidade Federal de São Carlos, São Carlos, São Paulo, Brasil.

Correspondência:

Verônica Clemente Ferreira.

Universidade Federal de Jataí, câmpus Jatobá, Cidade Universitária, BR 364, Km 195, nº 3800, Jataí, GO, Brasil. CEP: $75801-615$.

E-mail: verosocio@ufg.br

Recebido em 11/08/20; Aceito em 03/09/20. 


\section{INTRODUÇÃO}

A teoria social do gênero o define como algo dinâmico, relacional e dependente de fatores históricos, culturais e sociais'. Um olhar para o ser mulher como algo além da anatomia e, portanto, relacionado às estruturas sociais de poder é de suma importância para a evolução dos cuidados em saúde da mulher. Nessa perspectiva, as políticas em saúde, antes restritas à função reprodutiva, caminham no sentido de uma atenção integral, e a medicina passa a reconhecer como a desigualdade de gênero pode determinar diferenças nos processos de saúde, sofrimento e adoecimento².

No contexto da pandemia da coronavirus disease 2019 (Covid-19), o agravamento de desigualdades preestabelecidas torna ainda mais urgente a atenção às especificidades de populações vulnerabilizadas, incluindo as mulheres. Elas representam $70 \%$ da força de trabalho em saúde e são a maioria na linha de frente da responsabilidade pelos cuidados, sejam estes domésticos ou institucionais ${ }^{3}$. Ademais, com a mudança nas relações sociais, o isolamento e a crise econômica advindos da pandemia, houve um aumento da violência contra a mulher, pois as mulheres encontram-se confinadas com seus agressores ${ }^{4}$. A quarentena também tem dificultado o acesso à saúde, seja por causa das mudanças na dinâmica dos serviços, seja pela incerteza da mulher quanto à segurança em buscar atendimento. A preocupação se dá especialmente em relação ao grave aumento na mortalidade materna na pandemia e à dificuldade de acesso à saúde e aos direitos sexuais e reprodutivos, considerados essenciais durante crises sanitárias ${ }^{5,6}$.

É importante pontuar que as mulheres não são um grupo homogêneo7. Nesse sentido, uma análise interseccional, que leve em consideração idade, raça, sexualidade, deficiência, território, classe, entre outros condicionantes, faz-se essencial ${ }^{8}$.

Àluz do conceito ampliado de saúde ${ }^{9}$, é perceptível como, em uma sociedade patriarcal como a brasileira, a desigualdade no acesso a direitos básicos de acordo com o gênero impacta as condições de saúde. Reconhecendo esse fato, surgem, no Sistema Único de Saúde (SUS), as políticas públicas voltadas à atenção integral à mulher, e em 2005, é implementada a Política Nacional de Atenção Integral à Mulher (PNAISM).

O reconhecimento das vulnerabilidades sociais e de seus efeitos na saúde também implicou uma reorganização na educação médica. Assim, é prevista a educação médica em consonância com o conjunto de políticas do SUS, o que abrange a PNAISM. As atuais Diretrizes Curriculares Nacionais (DCN) para os cursos de Medicina incluem a consideração da diversidade de gênero, a integralidade e a humanização no atendimento como essenciais à formação ${ }^{10}$. Apesar dos avanços, é necessário reconhecer a educação como ferramenta que também sustenta as estruturas de poder. De acordo com
Hirsch", as instituições são: "modos de orientação, rotinização e coordenação de comportamentos que tanto orientam a ação social como a tornam normalmente possível". Ou seja, o grupo que pode dominar essas ferramentas é capaz de “institucionalizar seus interesses, impondo à sociedade regras, padrões de condutas e modos de racionalidade que tornem 'normal' e 'natural' o seu domínio”(p. 26)'2.

A atual conjuntura de pandemia, ao evidenciar desigualdades no acesso à saúde, alerta para o compromisso da educação médica com as necessidades de populações vulnerabilizadas, como as mulheres. O momento é desafiador, mas, ao mesmo tempo, instiga a ponderação sobre o cenário atual e novas possibilidades. O objetivo deste ensaio é refletir sobre as competências necessárias ao egresso da graduação em Medicina, tendo em vista a atenção integral à mulher.

\section{DESENVOLVIMENTO}

Saúde materna e infantil

As políticas de saúde materna e infantil da última década melhoraram os indicadores do desenvolvimento infantil e da mortalidade materna e infantil. Inserida no conjunto de políticas que coadunam com a PNAISM², a Rede Cegonha (RC), publicada pelo Ministério da Saúde (MS) em 2011, buscou organizar as condutas dos profissionais e gestores de saúde para garantir o direito de mulheres e crianças ao acolhimento, ao atendimento qualificado e ao acesso a informações, além de proporcionar condições para o parto seguro e para o desenvolvimento infantil saudável ${ }^{13,14}$. A dificuldade em tornar essas estratégias abrangentes em nível nacional se evidencia em tempos de pandemia da Covid-19 $9^{15,16}$. Estudos recentes sobre o desfecho dos casos de gestantes e puérperas com Covid-19 $9^{17-19}$ observaram que, desde 26 de fevereiro de 2020, 124 mulheres foram a óbito, cifra 3,4 vezes maior do que o total de mortes maternas por Covid-19 no resto do mundo ${ }^{17,18}$. A pandemia aumenta os obstáculos para o acesso aos cuidados do pré-natal e puerpério e aos serviços de maternidade, sobretudo para mulheres pobres e negras. Diabetes, doenças cardiovasculares e obesidade - fatores de risco evitáveis - surgiram como comorbidades associadas a 48,4\% dessas mortes ${ }^{17,18}$, apontando para a já problemática qualidade do atendimento no pré-natal e puerpério ${ }^{17-19}$. $O$ alto número de cesarianas no Brasil também é apontado como fator de explicativo, pelo risco do pós-operatório para mulheres infectadas1 $1^{7,18,20}$. O aumento da mortalidade materna mostra a dificuldade de acesso das mulheres aos cuidados intensivos (internação em unidade de terapia intensiva (UTI) e suporte de ventilação), revelando a escassez de recursos humanos e materiais em terapia intensiva nas maternidades ${ }^{17,18,20}$. Ademais, a falta de uma política de testagem massiva dessa população 
leva à subnotificação dos casos e dificulta a mensuração e o correto enfrentamento do problema ${ }^{17,18,20}$.

Nesse contexto, cabe refletir sobre o papel do/a médico/a e das competências desenvolvidas em sua formação. A leitura das condições de vida e de saúde das usuárias sob o enfoque das relações de gênero, conforme diretriz da $\mathrm{PNAISM}^{2}$, permite compreender as diversas nuances da condição feminina, geradoras de múltiplas situações de desigualdade e opressão.

A formação generalista deve possibilitar ao/à estudante de Medicina formar competências para o cuidado à saúde materna e infantil preconizado pelas políticas do SUS, de modo a garantir a integralidade do cuidado para as mulheres durante a gestação, o parto e o puerpério. De acordo com as DCN, cabe ao/à médico/a "promover estilos de vida saudáveis, conciliando as necessidades tanto dos seus clientes/pacientes quanto às de sua comunidade, atuando como agente de transformação social”" ${ }^{\prime \prime}$. 0 ensino médico deve desenvolver no/a educando/a competências de comunicação e de trabalho em equipe multiprofissional ${ }^{10}$. O/a egresso/a é pensado/a como um/a educador/a em saúde, apto/a a utilizar linguagem clara e coerente ao longo das consultas de pré-natal e pós-parto e orientar sobre aspectos relacionados à saúde materna e infantil. As competências comunicacionais são fundamentais para estimular as mulheres a apropriar-se do conhecimento. É importante que o/a estudante de Medicina aprenda a dialogar com a cultura das mulheres, partilhando informações de maneira corresponsável e não impositiva.

O/a estudante deve ser preparado/a para compor equipes multiprofissionais e participar de atividades de educação voltadas não apenas às mulheres, mas também a cônjuges, familiares e comunidades, envolvendo-os na rede de apoio. $\mathrm{O}$ aprendizado de técnicas apropriadas de comunicação ao longo da formação permite que o/a egresso/a seja capaz de dialogar com as gestantes sobre seus anseios e suas inseguranças quanto aos sinais do trabalho de parto e aos procedimentos hospitalares, compartilhando informações sobre o direito delas de ter suas escolhas respeitadas no momento do nascimento do bebê' ${ }^{13,14}$.

Um segundo leque de competências refere-se ao domínio de conhecimentos da natureza biopsicossocioambiental, à atuação ética e à capacidade de interpretar informações e resolver problemas da prática ${ }^{10}$. Aplicadas à saúde materna e infantil, essas demandam das escolas médicas desenvolverem no/a estudante a capacidade de obter informações sobre o território, a comunidade e o meio social das pacientes. A escuta atenta às mulheres, sem julgamentos ou preconceitos, é imprescindível para identificar situações de vulnerabilidade e/ou fragilização e para o encaminhamento aos setores adequados para atendê-las ${ }^{2,13,14}$. As DCN também preveem que o/a egresso/a deve manter-se atualizado/a com a legislação pertinente à saúde10, o que significa conhecer a legislação e os manuais de conduta que amparam o cuidado à saúde materna e infantil 2,13,2-25.

No campo da saúde materna e infantil, é importante que a formação generalista do/a estudante o/a muna de recursos semiológicos e conhecimentos sobre as mudanças do corpo feminino na gestação, no parto e no pós-parto, e sobre os exames necessários para cada fase. Isso possibilitará ao/à médico/a avaliar corretamente a situação de cada paciente, fazer a classificação de risco e tomar decisões corretas sobre a terapêutica a ser adotada ${ }^{24,25}$. Mais importante ainda é o aprendizado da utilização e interpretação da informação científica relacionada aos procedimentos no momento do parto, em vista da manutenção, nos serviços de atenção obstétrica brasileiros, do uso de práticas não amparadas por evidências e do número elevado de cesarianas, que provocam sofrimento desnecessário e implicam riscos para a mulher e para o bebêt, $\hat{2}^{2,13,2,22,26}$. Na pandemia da Covid-19, estes são potencializados para mulheres infectadas e/ou com comorbidades ${ }^{17,20}$, o que torna fundamental a preparação do/a aluno/a para conduzir tais situações na urgência e emergência.

\section{Saúde sexual}

Segundo a Organização Mundial da Saúde - OMS (2020) ${ }^{27}$,

[...] saúde sexual é um estado de bem-estar físico, mental e social em relação à sexualidade. Requer uma abordagem positiva e respeitosa da sexualidade $e$ das relações sexuais, bem como a possibilidade de ter experiências sexuais agradáveis e seguras, livres de toda coerção, discriminação e violência.

Nesse sentido, são objetivos da PNAISM a ampliação e qualificação da atenção clínico-ginecológica, a promoção da atenção às mulheres e adolescentes em situação de violência doméstica e sexual, e a prevenção e o controle das infecções sexualmente transmissíveis (IST) e da infecção pelo HIV na população feminina ${ }^{2}$. Entretanto, é preciso avançar na ampliação da cobertura da prevenção combinada, de exames, diagnósticos e tratamentos, desenvolvendo ações específicas para as mulheres em situação de maior vulnerabilidade às desigualdades de acesso à saúde ${ }^{28-30}$. Apesar das conquistas, a violência contra a mulher representa um problema de saúde pública, sendo a violência sexual o terceiro tipo mais comum ${ }^{28,31}$. A expansão da notificação dos casos de violência, a garantia de atendimento qualificado e humanizado à mulher em situação de violência e a sensibilização das equipes para as especificidades de grupos mais vulneráveis, como mulheres transgênero, permanecem como desafios ${ }^{28,30,32-34}$. $O$ isolamento social pela Covid-19 aumentou a violência doméstica contra 
as mulheres (inclusive trans, lésbicas e bissexuais), relacionada à maior vulnerabilidade delas à manipulação psicológica e ao controle das finanças, agravadas pelo maior consumo de álcool ou drogas ilícitas pelo agressor ${ }^{35-37}$. A diminuição de denúncias de violência sexual possivelmente se dá pelo menor acesso aos serviços de apoio (inclusive por receio de contrair a Covid-19) e pelo contato constante com o agressor ${ }^{35,36}$. Tal cenário sinaliza a necessidade de prepararo/a estudante para enfrentar as situações de violência, identificar seus sinais e acolher e desenvolver ações para a construção de redes de apoio às vítimas.

As políticas de saúde também invisibilizam outros aspectos da sexualidade, como: identidade de gênero, orientação sexual, sensualidade e relacionamento com o outro e com o próprio corpo ${ }^{38}$. A sexualidade da mulher é reprimida, sendo equiparada ora à reprodução ora ao perigo de IST e gravidez na adolescência, visão que perpetua a repressão do prazer sexual feminino e repercute em violência ${ }^{38,39}$. Isso se reflete nos serviços de saúde para mulheres, em sua maioria centrados em saúde reprodutiva ${ }^{38}$. Os/as profissionais não são preparados/as para atender às questões relacionadas à sexualidade, fato que se agrava quando o assunto é orientação sexual e identidade de gênero, afastando mulheres LGBTQUIA+ dos serviços de saúde 40-42. $^{2}$

As DCN de Medicina não abordam diretamente o conhecimento da saúde sexual da mulher como competência necessária ao/à egresso/ $\mathrm{a}^{10}$, embora contemplem o respeito às diferenças ao citarem que o/a profissional deverá considerar diversidades, como a de gênero, garantindo a universalidade e a equidade, sem privilégios nem preconceitos ${ }^{10}$. Entre habilidades e conteúdos curriculares listados nas DCN, encontram-se elementos que podem ser relacionados ao assunto.

O primeiro ponto a ser destacado concerne à "formação [...] humanista, crítica e reflexiva, [...] com responsabilidade social" ${ }^{\prime 0}$. O/a egresso/a deve compreender os determinantes sociais da saúde, incluindo as questões de gênero, assim como as vulnerabilidades no processo saúde-doença, e entrar em contato com conhecimentos acerca dos direitos humanos ${ }^{10}$. Essa compreensão deve ser estendida à educação em saúde sexual da mulher, que precisa sair do padrão de medicina centrada na doença e na prevenção e alcançar um modelo de bem-estar sexual, capacitando o/a graduando/a a lidar com a sexualidade da mulher com sensibilidade, entendendo-a como complexa e baseada em consentimento ${ }^{38}$.

O segundo ponto é referente à habilidade de comunicação, com empatia e interesse, praticando a escuta ativa e favorecendo a construção de vínculo10. Porém, os/ as médicos/as não se sentem à vontade para ouvir e falar sobre sexualidade, limitação mais evidente no atendimento às mulheres trans, em que a falta de clareza nas informações e o não acolhimento são as principais queixas ${ }^{42,43}$. Outra consequência é o receio, por lésbicas e mulheres bissexuais, de relatar a orientação sexual em uma consulta ${ }^{41}$.

A formação médica também necessita que se conheçam e se vivenciem as políticas públicas de saúde ${ }^{10}$. É essencial que os/as graduandos/as entrem em contato, sobretudo nos cenários de prática, com políticas de saúde da mulher, destacando-se a PNAISM e as políticas voltadas para a saúde de populações vulneráveis, como Lésbicas, Gays, Bissexuais, Travestis e Transexuais (LGBT), mulheres negras, mulheres com deficiência e mobilidade reduzida, mulheres do campo e da floresta, mulheres privadas de liberdade no sistema prisional e mulheres dos povos indígenas ${ }^{29,30}$.

Por fim, as habilidades de realizar anamnese e exame físico e definir diagnóstico e tratamento ${ }^{10}$ são limitadas pela falta de equipamentos e conhecimentos adequados para exame ginecológico de mulheres com baixa estatura, com deficiência e com mobilidade reduzida; e para indicar mamografia para mulheres trans em hormonização ${ }^{43,44}$.

\section{Direitos sexuais e reprodutivos}

No tocante à saúde sexual e reprodutiva, a crise sanitária descortinou e aprofundou antigos problemas, como: gravidez não planejada, acesso ao aborto seguro, elevada mortalidade materna ${ }^{45}$. Trata-se de problemas perpassados por determinantes sociais de saúde, notadamente classe, raça/cor e escolaridade ${ }^{46,47}$. Ou seja, a pandemia vem vulnerabilizando mais as pessoas já vulnerabilizadas na pré-pandemia: as mulheres, e, dentre elas, as mais pobres e as negras ${ }^{48}$.

Ainda que não se possam mensurar os efeitos da crise sanitária na saúde das mulheres, sabe-se que houve mudança na rede de serviços eletivos, essenciais e de emergência. É importante, mesmo durante este período, a garantia das consultas (por telemedicina ou presencial, se possível) e da oferta de métodos contraceptivos. Estima-se que a redução de $10 \%$ na oferta de métodos contraceptivos atingiria cerca de 48 milhões de mulheres no mundo com a estimativa de sete milhões de gestações não planejadas ${ }^{49}$.

A OMS declarou a necessidade de garantir o acesso e continuidade a métodos contraceptivos ${ }^{50}$. De acordo com a Comissão Interamericana de Direitos Humanos, devem ser garantidas, neste contexto de pandemia, a continuidade e disponibilidade dos serviços de saúde sexual e reprodutiva e aumentadas as ações de educação sexual integral, compartilhando as informações por meios acessíveis ${ }^{51}$.

A formação médica interfere diretamente na prescrição dos métodos contraceptivos, ainda bastante voltada para o controle dos corpos das mulheres ${ }^{52}$, e, consequentemente, a oferta e o uso de métodos contraceptivos de longa duração 
ainda são baixos ${ }^{53}$. Nesse sentido, torna-se importante, para propiciar o diálogo entre os sujeitos envolvidos com o cuidado, a construção de uma proposta educativa que não considere apenas o modelo biomédico, mas que também pondere problemáticas relativas ao gênero, à sexualidade, à autonomia e à liberdade. A formação profissional constitui a chave para a construção de práticas não discriminatórias e pautadas na integralidade da atenção, que garantam a promoção, a proteção e o exercício da sexualidade e da reprodução como direitos, e que sejam norteadoras das práticas assistenciais no âmbito da atenção à saúde ${ }^{54}$.

No campo do ensino, são necessárias práticas educativas e ações de cuidado pautadas nas tecnologias leves ${ }^{55}$. Em meio à pandemia, as tecnologias leves contribuirão na produção do cuidado a partir da compreensão do usuário quanto à sua singularidade; no estabelecimento de vínculos solidários e de

[...] participação coletiva no processo de gestão; no mapeamento e interação com as demandas sociais, coletivas e subjetivas de saúde; na valorização dos diferentes sujeitos implicados no processo de produção de saúde: usuários/as, trabalhadores/as e gestão; no fomento da autonomia e do protagonismo desses sujeitos; e no aumento do grau de corresponsabilidade na produção de saúde ${ }^{56}$.

A atenção à saúde sexual e reprodutiva na pandemia requer uma interação dialógica entre universidade e a sociedade. Para o desenvolvimento das habilidades supracitadas, a curricularização da extensão pode ser uma aliada por desafiar as escolas médicas a repensar a formação médica e as interfaces com a sociedade, no desenvolvimento de consciência cidadã em paralelo à formação técnica57.

\section{Cuidados destinados às mulheres no climatério}

De modo específico, questões relacionadas à garantia dos direitos sexuais e reprodutivos das mulheres foram tratadas na $12^{\mathrm{a}}$ Conferência Nacional de Saúde, em 2004. Na ocasião foi proposta a criação do programa especial de atenção ao climatério como política de saúde pública ${ }^{58}$.

O climatério é definido como uma fase biológica da vida, que envolve a transição entre o período reprodutivo e o não reprodutivo, segundo a OMS. A menopausa corresponde ao último ciclo menstrual, sendo confirmada após 12 meses de seu acontecimento, e ocorre normalmente dos 48 aos 50 anos de idade ${ }^{59}$. O climatério traz implicações sociais e concernentes ao envelhecimento, um período marcado de intensa ansiedade, medos e dificuldade social, decorrente das inúmeras sintomatologias apresentadas ${ }^{60}$.

Os medos criados nesse período são extremamente acentuados em tempos de pandemia e incertezas em relação ao corpo e aos aspectos psicossociais ${ }^{61}$. Assim, no atendimento à mulher climatérica ou menopausada, devem-se respeitar sua individualidade e o espaço social que influencia sua vida, em decorrência das manifestações variadas dessa transição. Além disso, muitas apresentam alguma patologia associada, reforçando a necessidade do esclarecimento, discussão e orientação do/a profissional médico/a $a^{62}$.

Na situação de pandemia da Covid-19, em que a oferta de serviços de saúde e o acesso a eles sofrem impacto negativo, mulheres climatéricas/menopausadas são negligenciadas do ponto de vista multidisciplinar e continuado das políticas de saúde. Com as limitações do atendimento na atenção primária, há risco de interrupção dos tratamentos com terapia de reposição hormonal para mulheres com sintomas do climatério, agravando tais sintomas e os fatores de risco para tromboembolismo (obesidade, tabagismo, diabetes, hipertensão, doenças do colágeno) e para efeitos adversos da Covid-19 nos sistemas de coagulação. Isso sinaliza a necessidade de o ensino médico reforçar no/a estudante as competências relativas ao conhecimento das transformações do corpo feminino no período e das diferentes terapêuticas (como reposição hormonal transdérmica ou abordagens menos agressivas e invasivas como a progesterona natural), contraindicações e limitações; ao exame físico adequado, à escuta e à avaliação individualizada de cada caso (sobretudo pacientes com Covid-19 internadas); assim como competências comunicacionais para estimular a manutenção de hábitos de vida saudáveis ${ }^{63}$.

Ao ensino médico cabe estimular no/a estudante a busca por conhecimentos cientificamente validados para decidir pela terapêutica mais indicada para cada mulher ${ }^{64}$. O Manual de atenção à mulher no climatério/menopausa, publicado em 2008 pelo Ministério da Saúde, detalha o cuidado necessário com essa população durante a transição de ciclo reprodutivo para não reprodutivo ${ }^{59}$, porém as $\mathrm{DCN}$ não apontam a atenção direcionada especificamente ao cuidado continuado. É fundamental que a escola médica ofereça oportunidades para que o/a estudante desenvolva competências relacionais e, uma vez egresso/a, possa acolher as pacientes climatéricas/ menopausadas, orientá-las adequadamente, propor-lhes os meios adequados e seguros para promover seu bem-estar e empoderá-las com conhecimentos que lhes permitam vivenciar o período com menos repercussões negativas em suas vidas ${ }^{58,61}$ e que promovam sua qualidade de vida nos âmbitos biológico e psicossocial.

\section{CONCLUSÃO}

Ao longo deste ensaio, observamos um conjunto de competências do/a médico visando ao desenvolvimento de um cuidado integral à saúde da mulher e em consonância 
com políticas públicas, orientações e diretrizes nacionais. Essenciais para o/a médico/a, seu desenvolvimento nos cursos de Medicina se torna necessário e urgente, sobretudo em cenários emergenciais, como a atual pandemia da Covid-19, que acentuam vulnerabilidades e dificultam o acesso a cuidados, levando a desfechos fatais.

Se, de acordo com as atuais DCN, os/as egressos/as devem estar aptos/as a atender seus/suas pacientes dentro dos mais altos padrões de qualidade e dos princípios da ética/bioética, a formação para a saúde da mulher deve ser capaz de conduzi-los/ as para ouvir as pacientes, respeitá-las em suas singularidades em cada fase do ciclo ginecológico e adotar um olhar ampliado para suas condições de vida, acolhendo-as e reconhecendoas como seres sociais, influenciadas pelo ambiente em que se inserem. A educação médica deve mobilizar no/a estudante competências culturais e atitudinais para desenvolver formas mais simétricas de relacionamento com as pacientes. A capacidade de comunicar-se adequadamente é fundamental para que o/a egresso/a atue como agente de transformação da vida das mulheres, promovendo ações que resultem em estilos de vida saudáveis e em maior nível de autonomia e controle destas sobre sua saúde, seu corpo, sua sexualidade e sua vida. $\mathrm{E}$ o estímulo ao conhecimento baseado em evidências e à priorização da segurança das pacientes permite desenvolver o profissionalismo no/a egresso, o que pode evitar desfechos trágicos, sobretudo num cenário de pandemia.

\section{AGRADECIMENTOS}

As autoras deste ensaio agradecem a todos/as os/as integrantes do grupo de trabalho Populações (In)Visibilizadas e Diversidades da Associação Brasileira de Educação Médica (Abem). Agradecem também a todas as pessoas que de alguma forma apoiam esta luta.

Verônica Clemente Ferreira agradece a todos/as os/as integrantes da equipe multidisciplinar o Projeto EU QUERO (Engaging Users for Quality Enhancement and Rights: Strengthening the maternal and child healthcare system over the first 1000 days in Brazil, pesquisa realizada através de uma parceria entre Universidade Federal de Jataí, Universidade Federal de Goiás, Universidade Federal do Maranhão e Universidade de Southampton, Reino Unido. Recebe apoio do Medical Research Council, do Reino Unido, e das Fundações de Amparo à Pesquisa do Estado de Goiás e do Estado do Maranhão - FAPEG e FAPEMA, como consta em https://caj. jatai.ufg.br/n/115446-projeto-eu-quero-parceria-entre-a-ufgrej-university-of-southampton-reino-unido-e-ufma) a reflexão coletiva e o aprendizado colaborativo, que lhe oportunizaram construir conhecimentos sobre saúde materna e infantil e sobre direitos em saúde.

\section{CONTRIBUIÇÃO DAS AUTORAS}

Verônica Clemente Ferreira foi responsável pela escrita e organização do ensaio. Mariana Regazzi Ferreira da Silva colaborou na redação e revisão do artigo. Elisa Hypólito Montovani colaborou na redação e revisão do artigo. Larissa Gobbi Colares colaborou na redação e revisão do artigo. Aridiane Alves Ribeiro colaborou na revisão geral e crítica do manuscrito. Natália Sevilha Stofel colaborou na redação, formatação e revisão do ensaio.

\section{CONFLITO DE INTERESSES}

As autoras declaram não haver conflito de interesses neste ensaio.

\section{REFERÊNCIAS}

1. Scott J. Gender: a useful category of historical analysis. In: Scott, J. Gender and the politics of history. New York: Columbia University Press; 1988. p. 28-50.

2. Brasil. Política Nacional de Atenção Integral à Saúde da Mulher: princípios e diretrizes. Brasília: Ministério da Saúde; 2004

3. Brito L, Borges L, Fortes P, Gomes A, Narciso L, Palácios M, et al. Impactos sociais da Covid-19: uma perspectiva sensível às desigualdades de gênero. Observatório Covid-19 Fiocruz; 2020 [acesso em 2 ago 2020]. Disponível em: https://www.arca.fiocruz.br/bitstream/icict/41375/2/ ImpactosSociais.PDF.

4. Câmara dos Deputados dos Brasil. Crescem denúncias de violência doméstica durante pandemia [acesso em 2 ago 2020]. Disponível em: www.camara.leg.br/noticias/661087-crescemdenuncias-de-violenciadomestica-durante-pandemia.

5. UNFPA Brasil. COVID-19: um olhar para gênero. Promoção da saúde e dos direitos sexuais e reprodutivos e promoção da igualdade de gênero. Março 2020 [acesso em 16 ago 2020]. Disponível em: https://www.unfpa.org/sites/ default/files/resource-pdf/Portoguese-covid19_olhar_genero.pdf.

6. Sociedade Brasileira de Medicina de Família e Comunidade. Nota sobre a exoneração de técnicas e da coordenadora de saúde das mulheres do Ministério da Saúde. Junho de 2020 [acesso em 29 jul 2020]. Disponível em: https://www.sbmfc.org.br/noticias/nota-sobre-a-exoneracao-de-tecnicase-da-coordenadora-de-saude-das-mulheres-do-ministerio-da-saude/.

7. Instituto Brasileiro de Geografia e Estatística. Estatísticas de gênero: indicadores sociais das mulheres no Brasil [acesso em 2 ago 2020]. Disponível em: https://biblioteca.ibge.gov.br/visualizacao/livros/liv101551 informativo.pdf.

8. Observatório Brasil da Desigualdade de Gênero. Saúde integral das mulheres, direitos sexuais e direitos reprodutivos [acesso em 2 ago 2020]. Disponível em: http://www.observatoriodegenero.gov.br/menu/ areas-tematicas/saude.

9. Brasil. Relatório final. 8 $8^{\text {a }}$ Conferência Nacional da Saúde; 17-21 mar 1986. Brasília: Ministério da Saúde; 1986.

10. Brasil. Resolução CNE/CES n 3, de 20 de junho de 2014. Institui Diretrizes Curriculares Nacionais do Curso de Graduação em Medicina e dá outras providências. [acesso em 16 ago 2020]. Disponível em: http://portal.mec. gov.br/index.php?option=com_docman\&view=download \&alias $=15874$ rces003-14\&category slug=junho-2014-pdf\&ltemid=30192.

11. Hirsch J. Forma política, instituições políticas e Estado - I. Crítica Marxista. 2007;(24);9-36. [acesso em 16 ago 2020]. Disponível em: https://www.ifch. unicamp.br/criticamarxista/arquivos_biblioteca/artigo212artigo1.pdf.

12. Almeida, S. Racismo estrutural. São Paulo: Loyola; 2020. p. 37-46.

13. Brasil. Portaria $n^{\circ} 1.459$, de 24 de junho de 2011. Institui, no âmbito do Sistema Único de Saúde - SUS - a Rede Cegonha [acesso em 3 ago 2020]. Disponível em: http://bvsms.saude.gov.br/bvs/saudelegis/gm/2011/ prt1459_24_06_2011.html. 
14. Ribeiro AA, Thomaz EF, De Paula HMM, Diehl DA, Tacsi YC, Ferreira, VC, et al. Projeto Eu Quero: cartilha de direitos das mulheres. Goiânia: Universidade Federal de Goiás; São Luís: Universidade Federal do Maranhão; 2019.

15. Instituto de Pesquisa Econômica Aplicada. Brasil revê metas globais de redução da mortalidade materna e na infância [acesso 5 ago 2020]. Disponível em: https://www.ipea.gov.br/portal/index.php?option=com_ content\&view=article\&id $=35013 \&$ catid $=10 \&$ Itemid $=9$.

16. Federação Brasileira de Ginecologia e Obstetrícia. Nota oficial de alerta às mortes maternas associadas à COVID-19. 18 jul 2020 [acesso em 4 ago. 2020]. Disponível em: https://www.febrasgo.org.br/pt/noticias/item/1067nota-oficial-de-alerta-as-mortes-maternas-associadas-a-covid-19.

17. Takemoto MLS, Menezes MO, Andreucci CB, Nakamura-Pereira M, Amorim MMR, Katz L, et al. The tragedy of COVID-19 in Brazil: 124 maternal deaths and counting. Int J Gynaecol Obstet. 2020 July 9 [acesso em 20 ago 2020]. Disponível em: https://www.arca.fiocruz.br/handle/ icict/42199.

18. Takemoto MLS, Menezes MO, Andreucci CB, Knobel R, Sousa LAR, Katz $L$, et al. Clinical characteristics and risk factors for mortality in obstetric patients with severe COVID-19 in Brazil: a surveillance database analysis. BJOG: Int J Obstet Gy. 2020;8(0):1-9 [acesso em 20 ago 2020]. Disponível em: https://obgyn.onlinelibrary.wiley.com/doi/abs/10.1111/1471-0528.16470.

19. Tomasi E, Fernandes PAA, Fischer T, Siqueira FCV, da Silveira DS, Thumé $\mathrm{E}$, et al. Qualidade da atenção pré-natal na rede básica de saúde do Brasil: indicadores e desigualdades sociais. Cad Saude Publica. 2017;33(3):e00195815 [acesso em 21 ago 2020]. Disponível em: http://www.scielo.br/scielo. php?script=sci_arttext\&pid=S0102-311X2017000305001\&lng=en.

20. Takemoto MSL, Menezes MO, Andreucci CB, Knobel R, Sousa LAR, Katz $L$, et al. Maternal mortality and COVID-19. The Journal of Maternal-Fetal $\&$ Neonatal Medicine. 2020,7(0):1-7 [acesso em 20 ago 2020]. Disponível em: https://www.tandfonline.com/doi/full/10.1080/14767058.2020.1786056.

21. Brasil. Atenção humanizada ao abortamento: norma técnica. 2a ed. Brasília: Ministério da Saúde; 2011 [acesso em 5 ago 2020]. Disponível em: https://bvsms.saude.gov.br/bvs/publicacoes/atencao_humanizada_ abortamento_norma_tecnica_zed.pdf.

22. Brasil. Lei $n^{\circ} 9.263$, de 12 de janeiro de 1996. Regula o $\S 7^{\circ}$ do art. 226 da Constituição Federal, que trata do planejamento familiar, estabelece penalidades e dá outras providências [acesso em 3 ago 2020]. Disponível em: http://www.planalto.gov.br/ccivil_03/leis/l9263.htm.

23. Brasil. Resolução ${ }^{\circ} 36$, de 3 de junho de 2008. Dispõe sobre Regulamento Técnico para Funcionamento dos Serviços de Atenção Obstétrica e Neonatal [acesso em 5 ago 2020]. Disponível em: http://bvsms.saude. gov.br/bvs/saudelegis/anvisa/2008/res0036_03_06_2008_rep.html.

24. Brasil. Diretrizes nacionais de assistência ao parto normal: versão resumida. Brasília: Ministério da Saúde; 2017 [acesso em 3 ago 2020]. Disponível em: http://bvsms.saude.gov.br/bvs/publicacoes/diretrizes_ nacionais assistencia parto normal.pdf.

25. Brasil. Manual de acolhimento e classificação de risco em obstetrícia. Brasília: Ministério da Saúde; 2014 [acesso em 3 ago 2020]. Disponível em: http://www.saude.gov.br/images/pdf/2014/maio/29/Manual-de-ACRem-Obstetricia-versao-26-de-maio--4-.pdf.

26. Leal MC, Pereira APE, Domingues RMSM, Theme Filha MM, Dias MAB, Nakamura-Pereira $M$, et al. Intervenções obstétricas durante o trabalho de parto e parto em mulheres brasileiras de risco habitual. Cad Saude Publica. 2014;30(Supl 1):17-32 [acesso em 5 ago 2020]. Disponível em: https://www.scielo.br/pdf/csp/v30s1/0102-311X-csp-30-s1-0017.pdf.

27. World Health Organization. Sexual health [acesso em 2 ago 2020]. Disponível em: https://www.who.int/topics/sexual health/en/.

28. Brasil. Documento de apoio às Conferências de Saúde das Mulheres Municipais, Regionais e Estaduais. Brasília: Ministério da Saúde; 2017.

29. Brasil. Política Nacional de Saúde Integral de Lésbicas, Gays, Bissexuais, Travestis e Transexuais (LGBT). Brasília: Ministério da Saúde; 2013.

30. Brasil. Política Nacional de Saúde Integral da População Negra. Brasília: Ministério da Saúde; 2017.

31. Brasil. Vigilância de violências e acidentes. Brasília: Ministério da Saúde; 2017.
32. Passos RL, Telles FSP, Oliveira MHB. Da violência sexual e outras ofensas contra a mulher com deficiência. Saúde Debate. 2019;43(4):154-64 [acesso em 8 ago 2020]. Disponível em: https://www.scielo.br/pdf/sdeb/ v43nspe4/0103-1104-sdeb-43-spe04-0154.pdf.

33. Zucchi EM, Barros CRS, Redoschi BRL, De Deus LFA, Veras MAS. Bemestar psicológico entre travestis e mulheres transexuais no Estado de São Paulo, Brasil. Cad Saude Publica. 2019;35(3):e00064618 [acesso em 8 ago 2020]. Disponível em: https://www.scielosp.org/pdf/csp/2019.v35n3/ e00064618/pt.

34. Grupo de Trabalho Marcha das Margaridas. Relatório final e plano de ação. Brasília: Grupo de Trabalho da Marcha das Margaridas; 2016.

35. Vieira PR, Garcia LP, Maciel ELN. Isolamento social e o aumento da violência doméstica: o que isso nos revela? Rev Bras Epidemiol. 2020;23:E200033 [acesso em 20 ago 2020]. Disponível em: https://www. scielo.br/pdf/rbepid/v23/1980-5497-rbepid-23-e200033.pdf.

36. Okabayashi NYT, Tassara IG, Casaca MCG, Falcão AA, Bellini MZ. Violência contra a mulher e feminicídio no Brasil - impacto do isolamento social pela COVID-19. Braz J Hea Rev. 2020;3(3):4511-31 [acesso em 20 ago 2020]. Disponível em: https://www.brazilianjournals.com/index.php/BJHR/article/ view/9998/8381.

37. Hafi B, Uvais NA. Difculties faced by sexual and gender minorities during COVID-19 crisis. Psychiatry Clin Neurosci. 2020;74(8):444 [acesso em 21 ago 2020]. Disponível em: https://onlinelibrary.wiley.com/doi/epdf/10.1111/ pcn.13080.

38. Bass TM. Exploring female sexuality: embracing the whole narrative. NCMJ. 2016;77(6):430-2 [acesso em 8 ago 2020]. Disponível em: https:// www.ncmedicaljournal.com/content/ncm/77/6/430.full.pdf.

39. Macintyre AKJ, Veja ARM, Sagbakken M. "Sexuality? Amillion things come to mind": reflections on gender and sexuality by Chilean adolescentes. Reprod Health Matters. 2015;23(46):85-95 [acesso em 8 ago 2020]. Disponível em: https://www.tandfonline.com/doi/pdf/10.1016/j. rhm.2015.11.003?needAccess=true.

40. World Health Organization. Brief sexuality-related communication: recommendations for a public health approach. Geneva: WHO; 2015.

41. Valadão RC, Gomes R. A homossexualidade feminina no campo da saúde: da invisibilidade à violência. Physis. 2011;21(4):1451-67 [acesso em 8 ago 2020]. Disponível em: https://www.scielosp.org/pdf/physis/2011. v21n4/1451-1467/pt.

42. De Oliveira I, Romanini M. (Re)escrevendo roteiros (in)visíveis: a trajetória de mulheres transgênero nas políticas públicas de saúde. Saúde Soc. 2020;29(1):e170961 [acesso em 9 ago 2020]. Disponível em: https://www.scielosp.org/pdf/sausoc/2020.v29n1/e170961/pt.

43. Brasil. Diretrizes de Atenção à Saúde das Mulheres com Deficiência e Mobilidade Reduzida. Brasília: Ministério da Saúde; 2018.

44. Sociedade Brasileira de Patologia Clínica, Sociedade Brasileira de Endocrinologia e Metabologia, Colégio Brasileiro de Radiologia e Diagnóstico por Imagem. Posicionamento Conjunto Medicina Diagnóstica inclusiva: cuidando de pacientes transgênero. Brasil: SBPC, SBEM, CBR; 2019

45. Brito L, Borges L, Fortes $\mathrm{P}$, Gomes $\mathrm{A}$, Narciso L, Palácios $\mathrm{M}$, et al. Impactos sociais da Covid-19: uma perspectiva sensível às desigualdades de gênero. Observatório Covid-19 Fiocruz; 2020 [acesso em 2 ago 2020]. Disponível em: https://www.arca.fiocruz.br/handle/icict/41375.

46. Miranda-Ribeiro P, Caetano AJ, Santos, TF. Saúde reprodutiva, sexualidade, raça/cor. Belo Horizonte: UFMG, Cedeplar; 2004.

47. Goes EF, Nascimento ER. Mulheres negras e brancas e os níveis de acesso aos serviços preventivos de saúde: uma análise sobre a desigualdade. Saúde Debate. 2013;37(99):571-9 [acesso em 20 ago 2020]. Disponível em: http://www.scielo.br/scielo.php?script=sci_ arttext\&pid=S0103-11042013000400004\&lng=es.

48. Insfran F, Muniz AGCR. Maternagem e Covid-19: desigualdade de gênero sendo reafirmada na pandemia. Diversitates International Journal. 2020;12(2):26-4. [acesso em 16 ago 2020]. Disponível em: http:// diversitates.uff.br/index.php/1diversitates-uff1/article/view/314. 
49. Portal de Boas Práticas e Saúde da Mulher, da Criança e do Adolescente. Principais questões sobre Covid-19 e planejamento reprodutivo [acesso em 13 set 2020]. Disponível em: https://portaldeboaspraticas.iff.fiocruz. br/atencao-mulher/principais-questoes-sobre-covid-19-e-planejamentoreprodutivo/.

50. World Health Organization. Anticoncepção/planejamento familiar e COVID-19 [acesso em 2 ago 2020]. Disponível em: https://www.who.int/ reproductivehealth/publications/emergencies/WHO-COVID-Q-and-Acontraception-por.pdf.

51. Comisión Interamericana de Derechos Humanos. Pandemia y derechos humanos en las Américas [acesso em 3 ago 2020]. Disponível em: https:// www.oas.org/es/cidh/decisiones/pdf/Resolucion-1-20-es.pdf.

52. Zorzanelli RT, Cruz MGA. O conceito de medicalização em Michel Foucault na década de 1970. Interface (Botucatu). 2018;22(66):721-31 [acesso em 3 ago 2020]. Disponível em: http://www.scielo.br/scielo.php?script=sci_ arttext\&pid=S1414-32832018000300721\&lng=en.

53. Farias MR, Leite SN, Tavares NUL, Oliveira MA, Arrais PSD, Bertoldi AD, et al. Utilização e acesso a contraceptivos orais e injetáveis no Brasil. Rev Saude Publica. 2016;50(Supl 1):14s. doi: 10.1590/s1518-8787.2016050006176.

54. Telo SV, Witt RR. Saúde sexual e reprodutiva: competências da equipe na atenção primária à saúde. Ciênc Saúde Colet. 2018;23(11):3481-90. doi: 10.1590/1413-812320182311.20962016.

55. Merhy EE, Franco TB. Por uma composição técnica do trabalho em saúde centrada no campo relacional e nas tecnologias leves. Apontando mudanças para os modelos tecnoassistenciais. Saúde Debate. 2003;27(65):316-24.

56. Ceccon RF, Schneider IJC. Tecnologias leves e educação em saúde no enfrentamento à pandemia da COVID-19. SciELO Public Health; 2020.
57. Almeida SMV, Barbosa LMV. Curricularização da extensão universitária no ensino médico: o encontro das gerações para humanização da formação. Rev Bras Educ Med. 2019; 43(1 Supl 1): 672-80. doi: 10.1590/1981-5271v43suplemento1-20190013.

58. Pereira QLC, Siqueira HCH. O olhar dos responsáveis pela política de saúde da mulher climatérica. Esc Anna Nery Rev Enferm [Internet]. 2009;2(13):366-71 [acesso em 8 ago 2020]. Disponível em: https://www. scielo.br/pdf/ean/v13n2/v13n2a18.pdf.

59. Ministério da Saúde. Manual de Atenção à Mulher no Climatério/ Menopausa. Brasília: Ministério da Saúde; 2008.

60. Aranha JS, Lima CB, Lima MNFA, Nobre JOC. Climatério e menopausa: percepção de mulheres usuárias da estratégia saúde da família. Temas em Saúde. 2016;16(2):588-612 [acesso em 8 ago 2020]. Disponível em: http://temasemsaude.com/wp-content/uploads/2016/08/16232.pdf.

61. Impactos Sociais da Covid-19: uma perspectiva sensível às desigualdades de gênero. Portal Fiocruz; 2020 [acesso em 8 ago 2020]. Disponível em: https://portal.fiocruz.br/documento/impactos-sociais-da-covid-19-umaperspectiva-sensivel-desigualdades-de-genero.

62. Kataguiri LG. O climatério no contexto da estratégia de saúde da família [monografia]. Belo Horizonte: Universidade Federal de Minas Gerais; 2009.

63. Valadares ALR, Rodrigues MAH. Terapia hormonal da menopausa em tempos de COVID-19. Sogimig; 2020 [acesso em 19 ago 2020]. Disponível em: http://www.sogimig.org.br/wpcontent/uploads/2020/04/Sogimig_Covid-19_ Reposicao-hormonal-da-menopausa_Para-medicos_Abr-2020.pdf.

64. Picoli RP, Domingo ALA, Santos SC, Andrade AHG, Araújo CAF, Kosloski RMM, et al. Competências propostas no currículo de medicina: percepção do egresso. Rev Bras Educ Med. 2017;41(3):364-71 [acesso em 8 ago 2020]. Disponível em: https://www.scielo.br/pdf/rbem/v41n3/0100-5502rbem-41-03-0364.pdf.

\section{ERRATA}

Na página 1:

Onde se lia:

"Elisa Hypólito Mantovani"”,

Leia-se:

"Elisa Hypólito Montovani"”

Revista Brasileira de Educação Média 44 (supl. 1): e147, 2020. 